\title{
Eritrodermia: estudo clínico-laboratorial e histopatológico de
} 170 casos *

\section{Erythroderma: a clinico-laboratorial and bistopathological study of 170 cases}

\author{
Nurimar C. Fernandes ${ }^{1}$ \\ Juan Piñeiro Maceira ${ }^{3}$ \\ Thaís Felix Leitão Rosa Dresch ${ }^{5}$
}

\author{
Fabíola de Souza e Mello Pereira ${ }^{2}$ \\ Tullia Cuzzi ${ }^{4}$ \\ Paula Pereira Araújo ${ }^{6}$
}

\begin{abstract}
Resumo: Fundamentos - A dermatite esfoliativa/eritrodermia é síndrome rara e grave, de etiologia freqüentemente desconhecida. A hospitalização é necessária para avaliação inicial e tratamento.

OвJETIV - Determinar o perfil clínico, laboratorial e histopatológico dos eritrodérmicos internados no HUCFF-UFRJ. MÉTODOS - No período de 1990 a 2007, 170 casos foram submetidos a hemograma, velocidade de hemossedimentação, bioquímica do sangue, provas de função hepática, proteínas totais e frações, pesquisa de células de Sézary no esfregaço de sangue periférico, radiografia de campos pleuropulmonares e biópsia de pele em três locais. RESULTADOS - Entre 92 homens e 78 mulheres, na faixa de 30 a 80 anos, identificaram-se 99 casos $(58,23 \%$ ) com dermatoses preexistentes; 37 (21,77\%) com farmacodermias; 18 (10,58\%) com linfomas cutâneos de células T e $16(9,4 \%)$ de causa indeterminada. Calafrios, prurido, linfonodomegalias e edema de membros inferiores destacaram-se dentre sinais e sintomas. O perfil laboratorial consistiu de aumento da VHS, eosinofilia, leucocitose, linfocitose e anemia. No grupo de etiologia indeterminada, dois padrões histológicos emergiram: dermatite psoriasiforme e dermatite inespecífica.

CoNCLUSÃo - A psoríase foi a causa mais freqüente; três biópsias de pele simultâneas podem aumentar a acurácia do diagnóstico histopatológico.

Palavras-chave: Dermatite esfoliativa; Dermatite esfoliativa/diagnóstico; Dermatite esfoliativa /etiologia; Psoríase
\end{abstract}

\begin{abstract}
BACKGROUND - Exfoliative dermatitis (erythroderma) is a rare, severe syndrome, frequently of unknown etiology. The hospitalization is required for the initial evaluation and to establish the treatment.

OBJECTNE - to determine the clinical, laboratorial and histologic profile of erythrodermic inpatients at HUCFF-UFRJ. MÉTHODS - in the period 1990-2007, 170 patients were submitted to complete blood cell count, erythrocyte sedimentation rate, blood urea, glucose and creatinine, liver function tests, proteinogram, circulating Sézary cells count, chest $X$ Rays and skin biopsy of three areas.

RESULTS - from 92 male and 78 female aging 30 to 80 years, a preexisting dermatosis was identified in 99 cases (58,23\%), reaction to internal drugs in 37 cases (21,77\%), T cell cutaneous lymphomas in 18 cases (10,58\%); in 16 cases (9,47\%), a causative factor could not be disclosed. Chills, pruritus, lymphadenopathy, lower limbs edema were the most freqüent signs/symptoms. Elevated erythrocyte sedimentation rate, eosinophilia, leukocytosis, peripheral blood lymphocytosis and anemia were the most common laboratory findings. Among the idiopathic cases, two histological patterns were noticed: psoriasiform and inespecific dermatitis.

CONCLUSION - the main cause of erythroderma was psoriasis; three simultaneous skin biopsies can enhance the accuracy of the histopathologic diagnosis.

Keywords: Dermatitis, exfoliative; Dermatitis, exfoliative/diagnosis, Dermatitis, exfoliative/etiology; Psoriasis
\end{abstract}

Recebido em 26.10.2007.

Aprovado pelo Conselho Consultivo e aceito para publicação em 29.09.2008.

* Trabalho realizado no Serviço de Dermatologia, Hospital Universitário Clementino Fraga Filho, Universidade Federal do Rio de Janeiro (UFRJ) - Rio de Janeiro (RJ), Brasil. Conflito de interesse: Nenhum / Conflict of interest: None

Suporte financeiro: Nenhum / Financial funding: None

Professora-associada da Faculdade de Medicina da Universidade Federal do Rio de Janeiro (UFRJ) - Rio de Janeiro (RJ), Brasil. Pós-graduada em dermatologia da Faculdade de Medicina da Universidade Federal do Rio de Janeiro (UFRJ) - Rio de Janeiro (RJ), Brasil. Professor adjunto da Faculdade de Medicina da Universidade Federal do Rio de Janeiro (UFRJ) - Rio de Janeiro (RJ), Brasil.

Professor adjunto da Faculdade de Medicina da Universidade Federal do Rio de Janeiro (UFRJ) - Rio de Janeiro (RJ), Brasil.

Pós-graduanda em dermatologia da Faculdade de Medicina da Universidade Federal do Rio de Janeiro (UFRJ) - Rio de Janeiro (RJ), Brasil.

Pós-graduanda em dermatologia da Faculdade de Medicina da Universidade Federal do Rio de Janeiro (UFRJ) - Rio de Janeiro (RJ), Brasil.

(C)2008 by Anais Brasileiros de Dermatologia 


\section{INTRODUÇÃO}

A eritrodermia/dermatite esfoliativa é síndrome que resulta de múltiplas causas e implica risco de vida nos idosos; está caracterizada por eritema e descamação generalizados $(\geq 80 \%$ ou $\geq 90 \%$ da superfície corporal). ${ }^{1}$

A despeito da variedade de desordens dermatológicas que podem levar à eritrodermia, a doença geralmente se inicia com placas eritematosas que em dias ou semanas estendem-se até o acometimento de quase toda a superfície cutânea. A descamação se inicia alguns dias após o eritema, principalmente em áreas flexoras. ${ }^{2}$ As escamas são geralmente brancas ou amareladas e finas, embora escamas argênticas espessas possam aparecer, particularmente em estádios agudos e em regiões palmoplantares. Com o progresso da descamação, a pele torna-se ressecada, recoberta por escamas lamelares finas, contudo, principalmente em vigência de colonização bacteriana, podem surgir escamas aderentes ou escamocrostas que exalam odor característico. Com o tempo, surge espessamento cutâneo associado ao edema e liquenificação e fissuras. ${ }^{1,2}$ No estádio crônico da doença observam-se alterações dos anexos: queda de cabelo difusa é descrita, assim como edema periorbital crônico, o qual pode resultar em enduração e perda de elasticidade da pele, levando a ectrópio e epífora. Uma variedade de alterações do aparelho ungueal é referida, incluindo espessamento da lâmina, ceratose subungueal, onicólise distal, pontos hemorrágicos, paroníquia, linhas de Beau, pittings e leuconíquia. A dermatite esfoliativa de longa data pode involuir deixando discromias residuais. ${ }^{3}$

As associações sistêmicas são comuns, como adenopatia axilar e inguinal, com linfonodos em geral moderadamente aumentados e de consistência firme, e hepatomegalia. ${ }^{4-11}$

A necessidade do repouso no leito sem exposição à luz solar, do uso liberado de emolientes, do aumento de ingesta protéica e de fluidos, bem como a rotina de investigação etiológica, são indicativos de internação.

Realizou-se estudo em eritrodérmicos na enfermaria de Dermatologia objetivando traçar seu perfil clínico-laboratorial e histopatológico.

\section{MATERIAL E MÉTODOS}

Estudo de desenho transversal em 170 pacientes eritrodérmicos internados no Hospital Universitário Clementino Fraga Filho no período de 1990 a 2007.

\section{Critérios de inclusão:}

- qualquer tempo de evolução; com/sem tratamento prévio;

- exame físico indicando sinais - manifestações obje- tivas reconhecíveis por intermédio de inspeção, percussão e ausculta - e sintomas como febre, sudorese, mialgia, calafrios e prurido;

- exame das mucosas visíveis;

- temperatura aferida na boca;

- hemograma, velocidade de hemossedimentação;

- bioquímica: dosagem de glicose, uréia e creatinina no sangue;

- proteínas totais e frações;

- enzimas hepáticas: TGO, TGP, fosfatase alcalina;

- bilirrubinas total/frações;

- pesquisa de células de Sézary em esfregaço de sangue periférico;

- radiografia de campos pleuropulmonares;

- biópsia de pele em três locais aleatórios com coloração pela hematoxilina eosina.

Critério de exclusão: não preenchimento dos requisitos acima definidos.

\section{Critérios clínicos e histológicos estabelecidos para definição das etiologias: ${ }^{1-11}$}

\section{Psoríase:}

- observação de escamas argênticas ou história de psoríase; placas em áreas extensoras; descamação acentuada no couro cabeludo; pitting nas 20 unhas; - alterações anatomopatológicas: paraceratose; neutrófilos na camada córnea; hipogranulose ou agranulose; acantose; adelgaçamento suprapapilar; alongamento de papilas; vasos dérmicos dilatados; infiltrado perivascular linfo-histiocitário.

\section{Eczema de contato:}

- temporalidade imediata entre aplicação da substância e surgimento dos sintomas; melhora do quadro com descontinuidade do uso;

- alterações anatomopatológicas: espongiose, eosinófilos na derme.

\section{Eczema atópico:}

- antecedentes e estigmas atópicos;

- alterações anatomopatológicas: espongiose; infiltrado dérmico, mononuclear perivascular.

\section{Eczema seborréico:}

- antecedentes de eritema e descamação em áreas seborréicas;

- alterações anatomopatológicas: paraceratose; acantose, espongiose, reação inflamatória perivascular.

Eritrodermia ictiosiforme congênita (não bolhosa): - presente ao nascimento; pele vermelha e descamativa; as escamas nos membros inferiores maiores $\mathrm{e}$ 
mais escuras; membranas mucosas poupadas; alterações ungueais;

- alterações anatomopatológicas: hiperceratose; acantose e paraceratose.

\section{Sarna crostosa:}

- história prévia positiva para escabiose comum; prurido moderado ou intenso com ou sem exacerbação noturna; lesões acinzentadas e ceratósicas entre dedos das mãos; pesquisa direta - em escamas de pele - positiva para Sarcoptes scabiei.

Pitiriase rubra pilar:

- pápulas foliculares, eritêmato-descamativas e pruriginosas; couro cabeludo, região frontal, tronco, membros superiores e inferiores; criança, adolescente e adulto;

- alterações anatomopatológicas: paraceratose focal e alternada; acantose; diminuição ou desaparecimento da camada granulosa; infiltrado linfo-histiocítico, perivascular.

\section{Drogas:}

- temporalidade entre início da medicação e surgimento dos sintomas; melhora com a suspensão da droga; precedendo o quadro: erupção máculo-pápulo-purpúrica, escarlatiniforme, líquen plano-símile ou urticariforme;

- alterações anatomopatológicas: alteração vacuolar dos queratinócitos basais; queratinócitos necróticos esparsos; infiltrado linfo-histiocitário liquenóide ou perivascular envolvendo a interface dermoepidérmica.

Linfoma cutâneo de célula T (LCCT):

- micose fungóide: história de longa data de quadros de eritrodermia intermitentes, infiltração de face, poiquilodermia, adenomegalias;

- síndrome de Sézary: distrofia ungueal, ceratose palmoplantar, adenomegalias; $\geq 10 \%$ de células de Sézary em sangue periférico;

- alterações anatomopatológicas: epidermotropismo formando microabscessos de Pautrier; infiltrado linfóide liquenóide ou perivascular em derme papilar; linfócitos de pequeno e médio tamanho com núcleos convolutos ou irregulares.

\section{RESULTADOS}

- A eritrodermia correspondeu a $1 \%$ dos pacientes hospitalizados com dermatoses. A idade no início do quadro de eritrodermia, excluindo as desordens hereditárias e eczema atópico, variou de 30 a 80 anos (média de idade: 53,5 anos); 92 (54,11\%) homens e 78 $(45,03 \%)$ mulheres. A progressão do quadro foi variável: dias (8\%), semanas (4\%) e meses (60\%) nos casos em que o tempo de evolução pôde ser explicitado. Em relação aos sinais e sintomas, prevaleceram calafrios (88/51,76\%), prurido (36/21,23\%), e linfonodomegalia (20/11,78\%) seguidos por febre (14/8,23\%) e edema de membros inferiores (12/7\%); artralgias e mialgias também foram referidos. A febre foi de amplitude intermediária (entre $37,6^{\circ} \mathrm{C}$ e $38,5^{\circ} \mathrm{C}$ ). Como a vasodilatação mascara a temperatura axilar $\left(35,5^{\circ} \mathrm{C}-37^{\circ} \mathrm{C}\right)$, o parâmetro foi temperatura oral (até $0,4^{\circ} \mathrm{C}$ maior que a axilar). Esses sinais e sintomas foram observados concomitantemente ou não. Exulcerações orais foram observadas em um caso.

Alterações ungueais incluíram pitting, ceratose subungueal, onicólise e onicorrexe.

Linfonodomegalias foram observadas associadas aos linfomas cutâneos de célula $\mathrm{T}$, às farmacodermias e à psoríase. As cadeias inguinais, seguidas de cadeias cervicais e axilares foram as de maior acometimento.

Em relação aos exames laboratoriais (Tabela 1) destaca-se o fato de que menos da metade dos casos mostrou alterações descritas na literatura. ${ }^{1}$

As alterações laboratoriais mais freqüentemente encontradas foram: velocidade de hemossedimentação aumentada, eosinofilia, leucocitose, anemia normocítica normocrômica, linfocitose, e elevação de TGO e TGP. Na maioria dos casos, bioquímica, proteínas totais e albumina se mantiveram dentro dos limites normais (Tabela 1). A radiografia de campos pleuropulmonares não evidenciou padrão que pudesse ser considerado específico da síndrome.

A casuística foi estratificada em: exacerbação de dermatose preexistente (psoríase, eczema de contato, eczema atópico, eczema seborréico, eritrodermia ictiosiforme congênita, sarna crostosa e pitiríase rubra pilar); hipersensibilidade às drogas; linfomas cutâneos de células T; sem definição etiológica (Tabela 2).

O fator causal identificado como o mais freqüente foi o de dermatoses preexistentes $(58,23 \%) \mathrm{e}$, entre elas, a psoríase (66 casos); em seguida, o eczema de contato (18 casos) às medicações - neomicina, bacitracina, coaltar, permanganato de potássio, benzoato de benzila - e chá de aroeira. O paciente com sarna crostosa era soropositivo (HIV); houve regressão da eritrodermia após tratamento da sarna crostosa. A literatura refere que a eritrodermia esfoliativa pode desenvolver-se em pacientes HIV-infectados durante a soroconversão., ${ }^{11}$

Trinta e sete casos $(21,77 \%)$ foram relacionados ao uso de medicações orais, agrupadas em antiinflamatórios não esteróides (Aines); anti-hipertensivos com radical sulfidrila (captopril, hidroclorotiazida, indapamida, clortalidona, furosemida); derivados da sulfonamida (sulfoniluréia, sulfona, sulfametoxazoltrimetoprim); antibióticos (amoxicilina, tetracilina); 
TABELA 1: Achados laboratoriais relevantes dos casos estudados

\begin{tabular}{|c|c|c|c|}
\hline Achados & & $\mathbf{N}^{\mathrm{o}}$ de pacientes & $\%$ \\
\hline Elevação da VHS & $>20 \mathrm{~mm} / \mathrm{h}$ & 65 & 38,23 \\
\hline \multirow[t]{5}{*}{ Hemograma } & Eosinofilia $>4 \times 10^{8} \mathrm{cel} / 1$ & 62 & 36,47 \\
\hline & Leucocitose $>10 \times 10^{9} \mathrm{cel} / 1$ & 61 & 35,88 \\
\hline & Homens-Hgb $<13 \mathrm{~g} / \mathrm{dl}$ & 34 & 20 \\
\hline & Mulheres-Hgb $<11,5 \mathrm{~g} / \mathrm{dl}$ & 15 & 8,8 \\
\hline & Linfocitose & 15 & 8,8 \\
\hline \multirow[t]{2}{*}{ Hepatograma } & Elevação de TGO & 15 & 8,8 \\
\hline & Elevação de TGP & 20 & 11,76 \\
\hline \multirow[t]{2}{*}{ Proteinograma } & Proteína total $<6$ & 12 & 7 \\
\hline & Albumina $<3$ & 8 & 4,7 \\
\hline Células de Sézary & $\geq 10 \%$ & 8 & 4,7 \\
\hline
\end{tabular}

* Dados coletados no HUCFF-UFRJ (1990-2007).

múltiplas drogas (carbamazepina, AAS, fluoxetina, clonazepan) em caso de uso simultâneo de drogas suspeitas.

Erupção escarlatiniforme, morbiliforme e erupção purpúrica foram observadas no grupo das drogas, precedendo o eritema e descamação.

Foram observados 18 (10,58\%) casos de LCCT, sendo 14 de micose fungóide e quatro de síndrome de Sézary.

Em 37 casos foi observado o aspecto histológico de dermatite psoriasiforme, sendo possível concluir o diagnóstico em 26 casos: eczema de contato (sete); drogas (seis); psoríase (cinco); eczema atópico (quatro); fotoeczema (dois). Micose fungóide foi diagnosticada em dois casos, através de biópsia de linfonodo que evidenciou linfócitos atípicos; em cinco casos com padrão histológico de dermatite inespecífica, não foi possível a correlação clínico-histológica (Tabela 2).

Nos casos com padrão de dermatite psoriasiforme houve, ou não, evidências para suspeição de causas referidas; foram observados camada granulosa preservada, paraceratose, acantose mais ou menos regular, alongamento das cristas epidérmicas com áreas de fusionamento focais e porções suprapapilares discretamente adelgaçadas; capilares dilatados, mas não tortuosos; infiltrado inflamatório de linfócitos, histiócitos, eosinófilos, neutrófilos e plasmócitos. ${ }^{1}$ A dermatite psoriasiforme ou proliferação epidérmica psoriasiforme é dermatite superficial, definida por hiperplasia epitelial caracterizada por alongamento uniforme das cristas interpapilares que tendem a se estender para baixo, isto é, para a derme. ${ }^{12}$

Nos casos com padrão de dermatite inespecífica foram observadas hiperceratose, paraceratose, acantose, espongiose esparsa e infiltrado inflamatório mononuclear perivascular. Não houve nesses casos relato de doença cutânea prévia ou ingestão de drogas suspeitas; não foram evidenciados estigmas atópicos e/ou antecedentes pessoais e familiares de atopia.

\section{DISCUSSÃO}

Em diferentes estudos são relatadas predominância do sexo masculino e média de idade de 60 anos. ${ }^{7,9}$ Nossos achados mostram proporção semelhante.

Há concordância na literatura quanto à maior prevalência das dermatoses preexistentes evoluindo para eritrodermia, em percentuais que variam de $27 \%$ a $66 \% .^{1-11}$ Neste estudo o percentual foi de 58,23\%, sendo a psoríase a dermatose mais freqüente.

Das condições dermatológicas, a psoríase é apontada como a causa mais comum., ${ }^{3,1314}$

Observou-se etiologia por drogas em 37 casos $(21,77 \%)$. A literatura relata eritrodermia por drogas em percentuais de 4 a $40 \%{ }^{1-11}$ A definição de droga causadora é difícil, pois freqüentemente há uso concomitante de vários medicamentos. A cronologia do surgimento da erupção, a história de quadro anterior e a freqüência do quadro em relação à droga utilizada são critérios importantes, uma vez que a reexposição à droga suspeita não é possível por motivos éticos. Em relação aos anti-hipertensivos, o captopril seria raramente causador de eritrodermia, ${ }^{15,16}$ porém principal agente de rash. Os bloqueadores de canal de cálcio também foram raramente relacionados com eritrodermia. ${ }^{17} \mathrm{O}$ captopril é inibidor da enzima conversora de angiotensina I em angiotensina II (ECA) e tem grupamento sulfidrila em sua composição. $O$ quadro surge em média um a quatro meses depois do início da droga, mas pode manifestar-se em poucos 
TABela 2: Distribuição dos casos estudados segundo etiologia

\begin{tabular}{|c|c|c|c|c|}
\hline Etiologia & Classificação & $\mathrm{N}^{\mathrm{o}}$ de $\operatorname{casos}$ & Totais & $\%$ \\
\hline \multirow[t]{7}{*}{ Dermatoses preexistentes } & Psoríase & 66 & & \\
\hline & Eczema de contato & 18 & & \\
\hline & Eczema atópico & 7 & & \\
\hline & Eczema seborréico & 3 & 99 & 58,23 \\
\hline & Eritrodermia ictiosiforme congênita & 3 & & \\
\hline & Sarna crostosa & 1 & & \\
\hline & Pitiríase rubra pilar & 1 & & \\
\hline \multirow[t]{5}{*}{ Drogas } & Múltiplas drogas & 20 & & \\
\hline & Anti-hipertensivos & 6 & & \\
\hline & Derivados da sulfa & 5 & 37 & 21,77 \\
\hline & Aines & 4 & & \\
\hline & Antibióticos & 2 & & \\
\hline \multirow[t]{2}{*}{ Linfomas cutâneos de células $\mathrm{T}$} & Micose fungóide & 14 & & \\
\hline & Síndrome de Sézary & 4 & 18 & 10,58 \\
\hline \multirow[t]{2}{*}{ Indeterminada } & Dermatite psoriasiforme & 11 & & \\
\hline & Dermatite inespecífica & 5 & 16 & 9,4 \\
\hline Total & & 170 & 170 & 99,98 \\
\hline
\end{tabular}

* Dados coletados no HUCFF-UFRJ (1990-2007).

Aines: antiinflamatórios não esteróides

dias; regride no prazo de seis semanas com sua suspensão ou com prednisona. ${ }^{18,21} \mathrm{O}$ enalapril foi descrito como desencadeador de eritrodermia em paciente psoriásico. $^{22}$

A hidroclorotiazida, a indapamida e a clortalidona são diuréticos tiazídicos que agem no túbulo distal do néfron diminuindo a reabsorção de sódio. ${ }^{19}$ A furosemida é um potente diurético que age na alça de Henle. Todas essas drogas são derivadas da sulfonamida. ${ }^{20}$ Sugere-se que pacientes com alergia aos derivados da sulfa evitem também hidroclorotiazida, clortalidona e furosemida pela possibilidade de reação cruzada; essa associação não é relatada para o captopril. ${ }^{21}$ Os tiazídicos observados nessa série não são citados em revisão recente.

Várias erupções por drogas que se apresentam morbiliformes, liquenóides ou urticariformes podem freqüentemente progredir para eritema e esfoliação. ${ }^{16}$ Em todos os pacientes com lesões purpúricas, a causa foi farmacodermia. Essas lesões foram identificadas mesmo na eritrodermia instalada. Predominaram em nosso estudo os casos em que havia uso simultâneo de drogas. Não se observou hipertermia em associação significativa com reação às drogas. ${ }^{23}$

O prurido não foi elemento identificador da possível causa, ao contrário da linfonodomegalia que, embora presente na farmacodermia e psoríase, o foi sobretudo nos casos de linfomas.
Em relação à presença da linfonodomegalia, alguns autores observam que a distribuição generalizada é mais associada às malignidades. ${ }^{5}$ Os linfomas cutâneos de células $\mathrm{T}$ constituem $25-40 \%$ dos casos de malignidades relacionados à eritrodermia. ${ }^{1}$ Nesta casuística ocorreu em 18 (10,58\%) dos casos. É referido que a contagem de células de Sézary circulantes superiores a $20 \%$ indicam síndrome de Sézary, porém percentuais inferiores a $10 \%$ constituem achado inespecífico na eritrodermia. ${ }^{1}$ Vale observar que a eritrodermia classifica o linfoma cutâneo de célula $\mathrm{T}$ - micose fungóide em T4; o percentual de células de Sézary atípicas circulantes em sangue periférico acima de 5\% em B1. No entanto, é prática corrente de muitos centros de referência de micose fungóide usar o critério de, no mínimo, $1.000 \mathrm{cel} / \mathrm{mm}^{3}$ para definição do comprometimento do sangue periférico. ${ }^{24}$

Uma proporção de casos $(9,4 \%)$ permaneceu sem etiologia definida contra percentuais referidos de 9 a 46\%. ${ }^{3,10}$ Para alguns autores ${ }^{25}$ a eritrodermia de etiologia indeterminada parece pertencer a um subgrupo de pacientes caracterizados por doença de longa evolução, difícil tratamento, ceratodermia palmoplantar, níveis elevados de IgE, eosinofilia de medula óssea e possível transformação maligna. Nos casos aqui mencionados a dosagem de IgE só foi realizada na suspeita de eczema atópico. Para outros 
autores, a eritrodermia de causa desconhecida e curso protraído pode ser secundária ao eczema atópico senil, bem como à ingestão de drogas mal apurada na anamnese ${ }^{1}$ o papel exato da IgE elevada na psoríase eritrodérmica não está definido. ${ }^{26}$

$\mathrm{Na}$ casuística anterior desse hospital, a psoríase foi identificada como a causa mais freqüente de eritrodermia esfoliativa $(51,4 \%) ; 22,8 \%$ dos casos permaneceram sem etiologia definida. ${ }^{27}$ No presente estudo (que não engloba casos anteriormente publicados) foram realizadas três biópsias de pele em cada paciente.

Definir a etiologia da eritrodermia é freqüentemente muito difícil, e os exames laboratoriais são de ajuda limitada. Múltiplas biópsias de pele em áreas representativas (dorso, membro superior, abdômen) devem ser feitas na tentativa de aumentar a possibilidade diagnóstica. ${ }^{28-30}$

\section{CONCLUSÕES}

A psoríase foi a causa mais freqüente; a correlação clínico-histopatológica na eritrodermia é difícil, pois os aspectos específicos da dermatose causadora são mascarados pelos inespecíficos da eritrodermia; a realização de três biópsias de pele simultâneas poderá aumentar a acurácia do diagnóstico histopatológico. 


\section{REFERÊNCIAS}

1. Sehgal VN, Srivastava G, Sardana K. Erythroderma/ exfoliative dermatitis: a synopsis. Int J Dermatol. 2004; $43: 39-47$

2. Sehgal VN, Srivastava G. Exfoliative dermatitis. A prospective study of 80 patients. Dermatologica. 1986;173:278-84.

3. Karakayli G, Beckham G, Orengo I, Rosen T. Exfoliative dermatitis. Am Fam Physician. 1999;59:625-30.

4. Abrahams I, MC Carthy JT, Sanders SL. Hundred one cases of exfoliative dermatitis. Arch Dermatol. 1963;87:96-101.

5. Nicolis GD, Helwig EB. Exfoliative dermatitis. A clinicopathologic study of 135 cases. Arch Dermatol. 1973;108:789-97.

6. Ndiaye B, Sissoko F, Strobel M, Marchand JP. Les érythrodermies de l' adulte (à propôs de 77 cas à Dakar). Dakar Med. 1979;24:65-74

7. Hasan T, Jansén TC. Erythroderma: a follow-up of fifty cases. J Am Acad Dermatol. 1983;8:836-40.

8. King LE, Dufresne G, Lovett GL, Rosin MA. Erythroderma: review of 82 cases. South Med J. 1986;79:1210-15.

9. Sigurdsson V, Toonstra J, Hezemans-Boer M, van Vloten WA. Erythroderma: a clinical and follow-up study of 102 patients, with special emphasis on survival. J Am Acad Dermatol. 1996; 35:53-7.

10. Pal S, Haroon TS. Erythroderma: a clinico-etiologic study of 90 patients. Int J Dermatol. 1998;37:104-7.

11. Fuchs BS, Sapadin AN, Phelps RG, Rudikoff D. Diagnostic dilemma: crusted scabies superimposed on psoriatic erythroderma in a patient with acquired immunodeficiency syndrome. Skinmed. 2007;6:142-4.

12. Elder DE, Elenitsas R, Johnson Jr B, Murphy GF, editors Lever's histopathology of the skin. 9th ed Philadelphia:Lippincot Williams \& Wilkins; 2005. p.75-138.

13. Boyd AS, Menter A. Erythrodermic psoriasis. Precipitating factors, course and prognosis in 50 patients. J Am Acad Dermatol. 1989;21(Pt 1):985-91.

14. Tomasini C, Aloi F, Solaroli C, Pippione M. Psoriatic erythroderma: a histopathologic study of forty five patients. Dermatology. 1997;194:102-6.

15. Norwood VF. Hypertension. Pediat Rev. 2002;23:197-208.

16. Solinger AM. Exfoliative dermatitis from captopril. Cutis 1982; 29:173-1.

17. Odeh M. Exfoliative dermatitis associated with diltiazem. J Toxicol Clin Toxicol. 1997;35:101-4.
18. Daniel F, Foix C, Barbet M, Schwebig A, Plouin F. Toxidermies au captopril: incidence au cours d'un traitement de 1321 mois/patients. Ann Dermatol Venereol. 1983;110:441-6.

19. Norwood VF. Hypertension. Pediat Rev. 2002;23:197-208.

20. Thestrup-Pedersen K. Adverse reactions in the skin from antihypertensive drugs. Dan Med Bull. 1987; 34 (Suppl1):3-5.

21. Gruchalla RS. Drug allergy. J Allergy Clin Immunol. 2003; 111(Suppl. 2):S548-59.

22. Antonov D, Grozdev I, Pehlivanov G, Tsankov N. Psoriatic erythroderma associated with enalapril. Skinmed 2006;5:90-2

23. Rym BM, Mourad M, Bechir Z, Dalenda E, Faika C, Iadh AM. Erythroderma in adults: a report of 80 cases. Int $\mathbf{J}$ Dermatol. 2005; 44:731-35.

24. Zinzani PL, Ferreri AJM, Cerroni L. Mycosis fungoides. Crit Rev Oncol Hematol. 2008;65:172-82.

25. Thestrup-Pedersen K, Halkier-Sørensen L, Søgaard H, Zackariae $H$. The red man syndrome. Exfoliative dermatitis of unknown etiology: a description and follow-up of 38 patients. J Am Acad Dermatol. 1988; 18:1307-12.

26. Li LF, Sujan SA, Yang H, Wang WH. Serum imunoglobulins in psoriatic erythroderma. Clin Exp Dermatol. 2005;30:125-7.

27. Wanke NCF, Souza FHC, Maceira J. Eritrodermia esfoliativa: estudo retrospectivo de 70 casos. An Bras Dermatol. 1990;65:181-5.

28. Milavec-Puretic V, Zoric Z, Zidanic M, Drcelic A, Stajminger G. Exfoliative dermatitis. Acta Dermatovenerol Croat. 2007;15:103-7.

29. Rothe MJ, Bernstein ML, Grantkeis JM. Life-threatening erythroderma: diagnosing and treating the red man. Clin Dermatol. 2005;23:206-17.

30. Jaffer AN, Brodell RT. Exfoliative dermatitis. Erythroderma can be a sign of a significant underlying disorder. Postgrad Med. 2005;117:49-51.

ENDEREÇO PARA CORRESPONDÊNCIA / MAILING ADDRESS: Nurimar C. Fernandes Rua Alexandre de Gusmão, $n^{\circ}$ 28, Apto. 201 20520120 - Rio de Janeiro RJ

Tel./Fax: (21) 2568-4158

E-mail: nurimarfernandes@terra.com.br

Como citar este artigo/How to cite this article: Fernandes NC, Pereira FSM, Maceira JP, Cuzzi T, Dresch TFLR, Araújo PP. Eritrodermia: estudo clínico-laboratorial e histopatológico de 170 casos. An Bras Dermatol.2008;83(6):526-32. 